TRABAJOS ORIGINALES

Rev Obstet Ginecol Venez. 2022; 82 (1): 47-58.

https://doi.org/10.51288/00820107

\title{
Factores asociados a dolores musculares y articulares en mujeres afrodescendientes climatéricas
}

\author{
Álvaro Monterrosa-Castro, ${ }^{1}$ (D) Geraldine Romero-Martínez, ${ }^{2}$ (D) Angélica Monterrosa-Blanco. ${ }^{3}$
}

\begin{abstract}
RESUMEN
Objetivo: Estimar la frecuencia de los dolores musculares y articulares e identificar si otros síntomas menopáusicos, la edad, la condición nutricional, el riesgo metabólico y el estado menopáusico, fueron factores asociados.

Métodos: Estudio transversal que hace parte del proyecto CAVIMEC (Calidad de vida en la menopausia y Etnias Colombianas), fue realizado en climatéricas afrodescendientes, edad entre 40-59 años y residentes en el Caribe colombiano. Fueron encuestadas en sus propias residencias, previo consentimiento informado. Se aplicó Menopause Rating Scale, herramienta que explora sintomas menopaúsicos. Se realizó regresión logística y se estimó el coeficiente de correlación de Spearman. Valor de $p<0,05$ fue estadisticamente significativo.

Resultados: Se estudiaron 420 mujeres. Edad: 50,0土6,1 años. El 22,8\% posmenopáusicas y el 82,8\% con obesidad abdominal; $243(57,9 \%)$ presentaban dolores musculares y articulares. Todos los síntomas menopaúsicos fueron más frecuentes entre las mujeres con dolores musculares y articulares: estado de ánimo depresivo (OR: 4,20/IC 95 \%: 1,55-11,74), edad 50-54 años (OR: 3,68/IC $95 \%: 1,53-8,82$ ), palpitaciones cardiacas (OR: 3,00/IC $95 \%: 1,51-5,98)$, edad 55-50 años (OR: 2,48/IC 95\%: 1,08$5,64)$ y oleadas de calor (OR: 1,92/IC 95\%: 1,11-3,31) se asociaron con dolores musculares y articulares. Igual se observó con el perímetro abdominal (OR: 0,322/IC 95\%: 0,233-0,405) y el perímetro de la cadera (OR: 0,318/IC 95\%: 0,229-0,401).

Conclusiones: Se observó elevada frecuencia de dolores musculares y articulares. El envejecimiento, síntomas psicológicos y somato/vegetativos, así como el perímetro abdominal y de la cadera, se asociaron significativamente con los dolores musculares $y$ articulares.
\end{abstract}

Palabras clave: Climaterio; Menopausia, Artralgia, Grupos Étnicos, Grupo de ascendencia continental Africana.

Factors associated with muscle and joint pain in climacteric Afro-descendant women SUMMARY

Objective: To estimate the frequency of muscle and joint pain and to identify if other menopausal symptoms, age, nutritional status, metabolic risk, and menopausal status were associated factors.

Methods: A Cross-sectional study which is part of the CAVIMEC project (Calidad de vida en la menopausia y Etnias Colombianas) was carried out in climacteric Afro-descendant women, between 40 and 59 years old, living in the Colombian Caribbean. They were surveyed in their own residences, prior informed consent. The Menopause Rating Scale was applied, this instrument assesses menopausal symptoms. Logistic regression was performed and the Spearman correlation coefficient was estimated. $P$ value $<0.05$ was statistically significant.

Results: 420 women were studied, median age $50.0 \pm 6.1,22.8 \%$ postmenopausal and $82.8 \%$ with abdominal obesity. 243 $(57.9 \%)$ had muscle and joint pain. All menopausal symptoms were more frequent among women with muscle and joint pain. Depressed mood OR: 4.20 (95\% CI:1.55-11.74), age 50-54 years OR: 3.68 (95\% CI:1.53-8.82), heart palpitations OR: 3.00 (95\% CI:1.51-5.98), age 55-50 years OR: 2.48 (95\% CI:1.08-5.64) and hot flashes OR: 1.92 (95\% CI:1.11-3.31) were associated with muscle and joint pain. The same was identified with the abdominal circumference OR: 0.322 (95 \% CI:0.2330.405) and hip circumference OR: 0.318 (95\% CI:0.229-0.401).

Conclusions: High frequency of muscle and joint pain was identified. Aging, psychological and somato/vegetative symptoms, as well as abdominal and hip circumference, were significantly associated with muscle and joint pain.

Keywords: Climacteric, Menopause, Arthralgia, Ethnic Groups, African Continental Ancestry Group.

${ }^{1}$ Médico. Especialista en Ginecología y Obstetricia. Líder Grupo de Investigación
Salud de la Mujer. Profesor titular. Facultad de Medicina. Universidad de Cartagena.
Colombia. ${ }^{2}$ Médico. Integrante Grupo de Investigación Salud de la Mujer. Facultad
de Medicina. Universidad de Cartagena. Colombia. ${ }^{3}$ Médico. Integrante Grupo de
Investigación Salud de la Mujer. Candidata a Magister en Epidemiología Clínica.
Fundación Universitaria Ciencias de la Salud (FUCS). Bogotá. Colombia. Correo
para correspondencia: alvaromonterrosa@gmail.com

Forma de citar este artículo: Monterrosa-Castro A, Romero-Martínez G, Monterrosa-Blanco A. Factores asociados a dolores musculares y articulares en mujeres afrodescendientes climatéricas. Rev Obstet Ginecol Venez. 2022; 82(1): 47-58. https://doi.org/10.51288/00820107

\section{INTRODUCCIÓN}

El climaterio es una de las etapas vitales de la mujer que se caracteriza por irregularidad en la liberación de las hormonas ováricas y síntomas psicobiológicos que potencialmente deterioran la calidad de vida (1). No obstante, pueden ser modulados por improntas 
genéticas, influencias nutricionales, sociales, culturales y étnicas (2). Hábitos, costumbres y otros elementos socio-antropológicos, contribuyen a explicar las diferencias en cuanto a frecuencia y severidad de los síntomas menopaúsicos, cuando se comparan diferentes etnias $(1,2)$.

Aunque los síntomas menopaúsicos más ampliamente evaluados son las oleadas de calor y el síndrome genitourinario de la menopausia, no son los únicos (3). La Menopause Rating Scale, es una herramienta especifica de calidad de vida en la menopausia, ampliamente utilizada a nivel mundial, traducida y validada en varios idiomas, que explora once síntomas menopáusicos, incluidos los dolores musculares y articulares (DMA) (4). Las artralgias, la artritis, los DMA y en general los dolores músculoesqueléticos, son más comunes en las mujeres, su frecuencia aumenta con la edad y al parecer muchos de los cuadros clínicos están asociados o guardan alguna relación fisiopatológica con el establecimiento de la menopausia (5).

Los síntomas de la menopausia experimentados por mujeres climatéricas afrodescendientes han sido menos explorados y descritos. Las comunidades afrodescendientes, son un grupo específico cuyos derechos humanos se deben promover y proteger. Por tal motivo, los años 2015 a 2024 fueron declarados por las Naciones Unidas como el Decenio Internacional de los Afrodescendientes, con el lema, "afrodescendientes: reconocimiento, justicia y desarrollo". Más de 200 millones de personas, que se identifican como descendientes de africanos, viven en América. Muchos millones más, viven en otras partes del mundo, fuera del continente africano (6).

Uno de cada cuatro latinoamericanos se identifica como afrodescendiente, actualmente existen más de 133 millones de personas, especialmente concentradas en Brasil, Colombia, Cuba, Venezuela, México y
Ecuador. Son comunidades heterogéneas, distribuidas desigualmente en la región, quienes comparten una larga historia de desplazamientos, vulnerabilidad, pobreza y exclusión (7). Colombia, es el segundo país con mayor población de afrodescendientes en América Latina y el Caribe. Se estima que 4671160 personas se reconocen como pertenecientes a dicho grupo poblacional, lo cual representa el 9,3\% de la población nacional, según el censo de población y vivienda del año 2018 (8).

A nivel mundial, especialmente en afrodescendientes, se ameritan más estudios que exploren los DMA, como un síntoma de la menopausia. Son escasos los datos en comunidades latinoamericanas que tienen influencias culturales y percepciones psicosociales que les son propias. La inclusión social de las afrodescendientes, incluye el derecho a que sean estudiadas científicamente, a que se identifiquen los síntomas que más les aquejan y a recibir recomendaciones que contribuyan con la sostenibilidad de su bienestar $(6,7)$. El objetivo fue estimar en mujeres climatéricas afrodescendientes, la frecuencia de los DMA e identificar si otros síntomas menopáusicos, la edad, la condición nutricional, el riesgo metabólico y el estado menopáusico, se comportaban como factores asociados.

\section{MÉTODOS}

Estudio transversal que hace parte del proyecto de investigación Calidad de Vida en la Menopausia y Etnias Colombianas (CAVIMEC), avalado por la Universidad de Cartagena, Colombia. Participaron mujeres que se autoreconocieron como afrodescendientes, que realizaban actividades laborales o domésticas y se consideraban saludables. Nacidas y residentes en municipios de la región caribe colombiana, reconocidos como asentamiento de comunidades con ancestros africanos traídos como esclavos en la época de la colonia, con edad entre 40 y 59 años, rasgos fenotípicos y color de piel negra, 


\section{FACTORES ASOCIADOS A DOLORES MUSCULARES Y ARTICULARES \\ EN MUJERES AFRODESCENDIENTES CLIMATÉRICAS}

hijas de padre y madre de dicha raza. Fueron invitadas a participar por encuestadoras, que las visitaron en sus residencias. Se excluyeron las embarazadas y las que tenían discapacidad psíquica o de lectoescritura, limitaciones para la deambulación, diagnóstico de patologías reumatológicas, antecedente de cirugía en las extremidades inferiores por lesiones congénitas o adquiridas.

Las mujeres participaron anónima y voluntariamente, recibieron información sobre la investigación y firmaron consentimiento informado, acorde con la Declaración de Helsinki y el Informe Belmont, que establecen principios éticos para la investigación en seres humanos. Se tuvieron en cuenta las Normas Científicas, Técnicas y Administrativas para la investigación en salud, establecidas en la resolución 8430-1993 del Ministerio de Salud de Colombia, que permite considerar el estudio como de riesgo mínimo. El proyecto fue aprobado por el comité de ética de la Universidad de Cartagena, Colombia, según Acta 117 de marzo de 2019.

Se aplicó un formulario impreso que contenía tres partes. La primera para registrar datos personales: edad, nivel educativo, estado civil, hábito de fumar, estado menopaúsico, antecedente de histerectomía u ooforectomía y diagnóstico de hipertensión arterial o diabetes. En la segunda se consignaba el peso, la estatura, el perímetro abdominal y de la cadera, medido por la encuestadora. La tercera parte incluía los interrogantes de la Menopause Rating Scale (MRS) para identificar once síntomas de la menopausia, incluidos los DMA. Cada ítem se responde como ausente, leve, moderado, severo o muy severo, asignando desde 0 hasta 4 puntos, respectivamente. Las últimas cuatro opciones de respuesta, se consideran presencia del síntoma (4). En una población menopaúsica colombiana se señaló alfa de Cronbach de 0,86 (9). Al final de cada semana se revisaban los formularios diligenciados. Los completos se enumeraban y legajaban en la carpeta estudios. Los incompletos también se enumeraban y se legajaban en la carpeta descartados. Para el grupo de mujeres incluidas en el estudio se encontró un valor de alfa de Cronbach de 0,81 para la escala MRS.

\section{Definiciones}

El estado menopaúsico se clasificó según Stages of Reproductive Aging Workshop (STRAW). Premenopausia: ciclos menstruales regulares o con variaciones inferiores a siete días. Transición a la menopausia: ciclos menstruales irregulares, con variaciones superiores a siete días o ausencia menstrual inferior a un año. Posmenopausia: doce o más meses de ausencia menstrual al aplicar el formulario (10). El estado nutricional se estableció según el índice de masa corporal calculado con el peso entre la estatura al cuadrado, acorde con clasificación universal indicada por la Organización Mundial de la Salud (OMS). Infrapeso $\left(\leq 18,49 \mathrm{~kg} / \mathrm{m}^{2}\right)$, normal $(18,50-24,99$ $\left.\mathrm{kg} / \mathrm{m}^{2}\right)$, sobrepeso $\left(25,00-29,99 \mathrm{~kg} / \mathrm{m}^{2}\right)$ y obesidad $\left(\geq 30,00 \mathrm{~kg} / \mathrm{m}^{2}\right)$. Perímetro abdominal $\geq$ a $89,00 \mathrm{~cm}$, fue indicador de obesidad abdominal, aplicando criterios propuestos para mujeres adultas colombianas (11). Además, el perímetro abdominal permitió establecer el nivel de riesgo metabólico: bajo $(<80 \mathrm{~cm})$, aumentado $(80-88 \mathrm{~cm})$ y muy aumentado $(>88 \mathrm{~cm})$, clasificación que también ha sido propuesta de la OMS, aunque menos conocida. El nivel de estudios se clasificó según años de escolaridad: primario (uno a cinco), secundario (seis a doce) y superior (trece o más).

\section{Tamaño de la muestra}

El censo poblacional colombiano de 2005, cuyos datos globales y proyecciones a la siguiente década, fueron informados por el Departamento Nacional de Estadística (DANE), estimó que para el año 2019, las mujeres residentes en Colombia con 40-59 años de edad eran 4347 590. Además, indicó que el 10,6 \% de la población colombiana se autoreconocía afrodescendiente, estando el $32,0 \%$ asentado en 
los departamentos geográficos de la región caribe. El 73,0 \% residían en áreas urbanas y el $27,0 \%$ en rurales. Basado en lo anterior se considera que 592514 son las mujeres colombianas con edad entre 40-59 años que se autoreconocen como afrodescendientes y 189605 las que residen en la región caribe, estas últimas son el universo del estudio. Un tamaño de la muestra de 384 mujeres fue calculado con un programa para análisis epidemiológico y estadístico (EPIDAT-3.1), definiendo nivel de confianza del $95 \%$, proporción esperada del $50 \%$, significancia del $5 \%$ y precisión absoluta del $5 \%$. Se agregó el $10 \%$ para compensar mujeres que se rehusaron a participar y $20 \%$ para reemplazar formularios descartados. Se tuvieron disponibles 506 formularios que se aplicaron diariamente, según el tamaño poblacional de los departamentos geográficos y la distribución urbano/ rural. A conveniencia no se incluyeron asentamientos cerrados, comunidades protegidas, circunscripciones especiales ni resguardos.

\section{Análisis estadístico}

La información de los formularios impresos de la carpeta estudios, fueron tabulados en una base de datos de Microsoft Excel 2007@. La población se subdividió en dos grupos, según la respuesta al ítem número once de la escala MRS: mujeres sin DMA y mujeres con DMA, fuesen leves, moderados, severos o muy severos. El análisis estadístico se realizó con Epi-Info-7 (Centers for Disease Control and Prevention, Atlanta, EEUU). Los datos continuos se expresan en medias y desviación estándar. Los categóricos en absolutos y porcentuales con intervalos de confianza al $95 \%$ (IC $95 \%$ ). Las significancias de las diferencias entre datos continuos, se estimaron con ANOVA o Mantel Haenszel, de acuerdo a la distribución de la varianza según el test de Bartlett. Las diferencias porcentuales se evaluaron con $\chi^{2}$. Se realizó regresión logística no ajustada y ajustada. DMA fue la variable dependiente y las covariables: rangos etarios, estados menopaúsicos y nutricionales, síntomas menopaúsicos, hábito de fumar, riesgo metabólico, obesidad abdominal y antecedentes médicos y quirúrgicos. La medida de asociación fue el OR con IC $95 \%$. Además, se estimó el coeficiente de correlación de Spearman entre DMA con las variables cuantitativas. Un valor de $p<0,05$ fue considerado estadísticamente significativo.

\section{RESULTADOS}

Se aplicaron 506 formularios, 86 (16,9 \%) fueron descartados y se realizó el estudio en 420 mujeres, $9,4 \%$ por encima del tamaño de la muestra. La edad promedio fue 50 años, el $22,8 \%$ posmenopáusicas, $82,8 \%$ con obesidad abdominal, $65,7 \%$ con riesgo metabólico muy aumentado, 20,0 \% fumadoras y $30,7 \%$ con hipertensión arterial crónica; 243 (57,9\%) informaron DMA. Se observó mayor edad, número de hijos, peso, perímetro abdominal, índice de masa corporal (IMC), sobrepeso, riesgo metabólico muy aumentado, obesidad abdominal, diabetes mellitus e hipertensión arterial, entre las mujeres con DMA $(\mathrm{p}<0,05)$ (Tablas 1 y 2$)$.

Todos los síntomas menopaúsicos fueron significativamente más frecuentes en las mujeres con DMA, excepto los problemas urinarios (tabla 3). Nueve síntomas menopáusicos, la transición a la menopausia, la posmenopausia, el sobrepeso, la obesidad, la hipertensión arterial, la diabetes y los rangos etarios superiores, se asociaron con DMA $(\mathrm{p}<0,05)$ (Tabla 4). Estado civil, años de estudio, hábito de fumar, antecedente de histerectomía u ooforectomía y obesidad abdominal, no se asociaron con DMA. En un modelo de regresión logística ajustado: el estado de ánimo depresivo, las palpitaciones cardiacas, las oleadas de calor y la edad superior a 50 años, se asociaron con DMA $(\mathrm{p}<0.05)$ (Figura 1). El perímetro abdominal, perímetro de cadera, edad, peso, el IMC y el índice cintura/cadera, se correlacionaron positiva y débilmente con DMA $(\mathrm{p}<0,001)$ (Tabla 5). 


\section{FACTORES ASOCIADOS A DOLORES MUSCULARES Y ARTICULARES EN MUJERES AFRODESCENDIENTES CLIMATÉRICAS}

Tabla 1. Características sociodemográficas. Variables cuantitativas

\begin{tabular}{|c|c|c|c|c|}
\hline \multirow[t]{2}{*}{ Variables } & $\begin{array}{c}\text { Todas } \\
\mathrm{n}=420\end{array}$ & $\begin{array}{c}\text { Sin dolores mus- } \\
\text { culares y articu- } \\
\text { lares } \\
n=177(42,1 \%)\end{array}$ & $\begin{array}{c}\text { Con dolores } \\
\text { musculares y } \\
\text { articulares } \\
\mathrm{n}=243(57,9 \%)\end{array}$ & \multirow[t]{2}{*}{$\mathrm{p}^{*}$} \\
\hline & \multicolumn{3}{|c|}{$\mathrm{X} \pm \mathrm{DS}$} & \\
\hline Edad, años & $50,0 \pm 6,1$ & $48,7 \pm 6,2$ & $51,2 \pm 5,8$ & $<0,001$ \\
\hline Años de estudio & $5,7 \pm 3,6$ & $5,9 \pm 3,6$ & $5,6 \pm 3,6$ & 0,45 \\
\hline Peso, kg & $66,1 \pm 12,2$ & $63,8 \pm 11,4$ & $67,9 \pm 12,5$ & $<0,001$ \\
\hline Perímetro abdominal, $\mathrm{cm}$ & $94,5 \pm 14,8$ & $90,0 \pm 14,1$ & $97,8 \pm 14,5$ & $<0,001$ \\
\hline Perímetro de cadera, $\mathrm{cm}$ & $102,63 \pm 14,42$ & $98,00 \pm 13,76$ & $106,00 \pm 13,97$ & $<0,001$ \\
\hline Índice cintura/cadera & $0,92 \pm 0,05$ & $0,91 \pm 0,05$ & $0,92 \pm 0,06$ & 0,55 \\
\hline Índice masa corporal, $\mathrm{kg} / \mathrm{m}^{2}$ & $25,4 \pm 3,8$ & $24,7 \pm 5,1$ & $25,9 \pm 4,9$ & 0,01 \\
\hline
\end{tabular}

*ANOVA.

Tabla 2. Características sociodemográficas. Variables cualitativas

\begin{tabular}{|c|c|c|c|c|c|}
\hline \multirow{2}{*}{\multicolumn{2}{|c|}{ Variables }} & Todas $\mathrm{N}=420$ & $\begin{array}{l}\text { Sin dolores musculares y } \\
\text { articulares } n=177(42,1 \%)\end{array}$ & $\begin{array}{l}\text { Con dolores musculares y } \\
\text { articulares } n=243(57,9 \%)\end{array}$ & \multirow[t]{2}{*}{$\mathrm{p} \S$} \\
\hline & & \multicolumn{3}{|c|}{$\mathrm{n}(\%)(\mathrm{IC} 95 \%)$} & \\
\hline \multicolumn{2}{|l|}{ Premenopausia } & $72(17,1)(13,8-21,0)$ & $45(25,4)(19,1-32,5)$ & $27(11,1)(7,4-15,7)$ & $<0,001$ \\
\hline \multicolumn{2}{|c|}{ Transición a la menopausia } & $252(60,0(55,2-64,5)$ & $100(56,5)(48,8-63,9)$ & $152(62,5)(56,1-68,6)$ & 0,21 \\
\hline \multicolumn{2}{|l|}{ Posmenopausia } & $96(22,8)(19,1-21,1)$ & $32(18,8)(12,7-24,5)$ & $64(26,3)(20,9-32,3)$ & 0,04 \\
\hline \multirow{4}{*}{ Rango etario } & $40-44$ & $101(24,1)(20,2-28,4)$ & $62(35,0)(28,0-42,5)$ & $39(16,0)(11,6-21,2)$ & $<0,001$ \\
\hline & $45-49$ & $92(21,9)(18,2-26,1)$ & $42(23,3)(17,6-30,6)$ & $50(20,5)(15,6-26,2)$ & 0,44 \\
\hline & $50-54$ & $93(22,1)(18,4-26,3)$ & $25(14,1)(9,3-20,1)$ & $68(27,9)(22,4-34,0)$ & $<0,001$ \\
\hline & $55-59$ & $134(31,9)(27,6-36,5)$ & $48(27,1)(20,7-34,3)$ & $86(35,3)(29,3-41,7)$ & 0,07 \\
\hline \multirow{2}{*}{ Estado civil } & Soltera/no unidas & $105(25,0)(21,1-29,3)$ & $44(24,8)(18,6-31,9)$ & $61(25,1)(19,7-31,0)$ & 0,95 \\
\hline & Casada/ unidas & $287(68,3)(63,7-72,6)$ & $124(70,0)(62,7-76,7)$ & $163(67,0)(60,7-72,9)$ & 0,51 \\
\hline \multirow{3}{*}{ Nivel de estudio } & Primario & $231(55,0)(50,2-59,6)$ & $94(53,1)(45,4-60,6)$ & $137(56,3)(49,8-62,7)$ & 0,50 \\
\hline & Secundario & $176(41,9)(37,2-46,6)$ & $76(42,9)(35,5-50,5)$ & $100(41,1)(34,9-47,6)$ & 0,71 \\
\hline & Superior & $13(3,1)(1,8-5,2)$ & $7(3,9)(1,6-7,9)$ & $6(2,4)(0,9-5,3)$ & 0,38 \\
\hline \multirow{4}{*}{ Estado nutricional } & Normal & $181(43,1)(38,4-47,8)$ & $91(51,4)(43,8-58,9)$ & $90(37,0)(30,9-43,4)$ & 0,003 \\
\hline & Sobrepeso & $159(37,8)(33,3-42,5)$ & $61(34,4)(27,4-41,9)$ & $98(40,3)(34,1-46,7)$ & 0,22 \\
\hline & Obesidad & $55(13,1)(10,2-16,6)$ & $15(8,4)(4,8-13,5)$ & $40(16,4)(12,0-21,7)$ & 0,01 \\
\hline & Infrapeso & $25(5,9)(4,0-8,6)$ & $10(5,6)(2,7-10,1)$ & $15(6,1)(3,5-9,9)$ & 0,82 \\
\hline \multirow{3}{*}{ Riesgo metabólico† } & Bajo & $72(17,1)(13,8-21,0)$ & $43(24,2)(18,1-31,3)$ & $29(11,9)(8,1-16,6)$ & $<0,001$ \\
\hline & Aumentado & $72(17,1)(13,8-21,0)$ & $36(20,3)(14,6-27,0)$ & $36(14,8)(10,6-19,9)$ & 0,13 \\
\hline & Muy aumentado & $276(65,7)(61,0-70,0)$ & $98(55,3)(47,7-62,8)$ & $178(73,2)(67,2-78,7)$ & $<0,001$ \\
\hline \multirow{3}{*}{ Hábito de fumar } & Actual & $84(20,0)(16,4-24,0)$ & $43(24,2)(18,1-31,3)$ & $41(16,8)(12,3-22,1)$ & 0,06 \\
\hline & Antes & $56(13,3)(10,4-16,9)$ & $16(9,0)(5,2-14,2)$ & $40(16,4)(12,0-21,7)$ & 0,02 \\
\hline & Nunca & $280(66,6)(62,0-71,0)$ & $118(66,6)(59,2-73,5)$ & $162(66,6)(60,3-72,5)$ & 1,00 \\
\hline \multicolumn{2}{|c|}{ Obesidad abdominal $\dagger$} & $348(82,8)(78,9-86,1)$ & $134(75,7)(68,7-81,8)$ & $214(88,0)(83,3-91,8)$ & $<0,001$ \\
\hline \multicolumn{2}{|l|}{ Histerectomía } & $29(6,9)(4,8-9,7)$ & $10(5,6)(2,7-10,1)$ & $19(7,8)(4,7-11,9)$ & 0,38 \\
\hline \multicolumn{2}{|l|}{ Ooforectomía bilateral } & $8(1,9)(0,97-3,7)$ & $2(1,1)(0,1-4,0)$ & $6(2,4)(0,9-5,3)$ & 0,10 \\
\hline \multicolumn{2}{|l|}{ Diabetes mellitus } & $46(10,9)(8,3-14,3)$ & $8(4,5)(1,9-8,7)$ & $38(15,6)(11,3-20,8)$ & $<0,001$ \\
\hline \multicolumn{2}{|l|}{ Hipertensión arterial } & $129(30,7)(26,4-35,2)$ & $38(21,4)(15,6-28,2)$ & $91(37,4)(31,3-43,8)$ & $<0,001$ \\
\hline
\end{tabular}

$\S$ Chi-cuadrado. $†$ Establecido por el perímetro abdominal. 
A MONTERROSA-CASTRO ET AL.

Tabla 3. Síntomas menopaúsicos. Menopause Rating Scale

\begin{tabular}{lcccc}
\hline Variables & \multicolumn{1}{c}{$\begin{array}{c}\text { Todas } \\
\mathrm{n}=420\end{array}$} & $\begin{array}{c}\text { Sin dolores } \\
\text { musculares y } \\
\text { articulares } \\
\mathrm{n}=177(42,1 \%)\end{array}$ & $\begin{array}{c}\text { Con dolores } \\
\text { musculares y } \\
\text { articulares } \\
\mathrm{n}=243(57,9 \%)\end{array}$ & $\mathrm{p}$ * \\
\cline { 2 - 5 } & $251(59,7)(55,0-64,3)$ & $69(38,9)(31,7-46,5)$ & $182(74,9)(68,9-80,2)$ & $<0,001$ \\
\hline Oleadas de calor & $148(35,2)(30,8-39,9)$ & $22(12,4)(7,9-18,1)$ & $126(51,8)(45,3-58,2)$ & $<0,001$ \\
Palpitaciones cardiacas & $150(35,7)(31,2-40,4)$ & $27(15,2)(10,3-21,4)$ & $123(50,6)(44,1-57,0)$ & $<0,001$ \\
Problemas para dormir & $112(26,6)(22,6-31,0)$ & $7(3,9)(1,6-7,9)$ & $105(43,2)(36,8-49,6)$ & $<0,001$ \\
Estado de ánimo depresivo & $88(20,9)(17,3-25,1)$ & $8(4,5)(1,9-8,7)$ & $80(32,9)(27,0-39,2)$ & $<0,001$ \\
Irritabilidad & $84(20,0)(16,4-24,0)$ & $11(6,2)(3,9-12,3)$ & $73(30,0)(24,3-36,2)$ & $<0,001$ \\
Ansiedad & $82(19,5)(16,0-23,5)$ & $13(7,3)(3,9-12,3)$ & $69(28,4)(22,8-34,5)$ & $<0,001$ \\
Agotamiento físico/mental & $28(6,6)(4,6-9,4)$ & $2(1,1)(0,1-4,0)$ & $26(10,7)(7,1-15,2)$ & $<0,001$ \\
Problemas sexuales & $90(21,4)(17,7-25,6)$ & $30(16,9)(11,7-23,0)$ & $60(24,6)(19,4-30,6)$ & 0,05 \\
Problemas urinarios & $17(4,05)(2,5-6,3)$ & $1(0,5)(0,0-3,1)$ & $16(6,58)(3,8-10,4)$ & 0,002 \\
Sequedad vaginal & & & &
\end{tabular}

(*) Chi-cuadrado

Tabla 4. Factores asociados a molestias musculo articulares. Regresión logística no ajustada

\begin{tabular}{lcc}
\hline Variables & OR (IC95\%) & $\mathrm{p}$ \\
\hline Sequedad vaginal & $12,39(1,62-94,35)$ & $<0,05$ \\
Problemas sexuales & $10,48(2,45-44,77)$ & $<0,001$ \\
Irritabilidad & $10,36(4,85-22,12)$ & $<0,001$ \\
Palpitaciones cardiacas & $7,58(4,54-12,66)$ & $<0,001$ \\
Ansiedad & $6,47(3,31-12,63)$ & $<0,001$ \\
Problemas para dormir & $5,69(5,52-9,20)$ & $<0,001$ \\
Agotamiento físico/mental & $5,00(2,66-9,38)$ & $<0,001$ \\
Oleadas de calor & $4,66(3,07-7,09)$ & $<0,001$ \\
Rango etario 50-54 con respecto a 40-44 & $4,32(2,35-7,94)$ & $<0,001$ \\
Diabetes mellitus & $3,91(1,77-8,62)$ & $<0,001$ \\
Posmenopausia vs premenopausia & $3,33(1,76-6,31)$ & $<0,001$ \\
Estado de ánimo depresivo & $3,27(1,23-8,71)$ & $<0,05$ \\
Rango etario 55-59 con respecto a 40-44 & $2,84(1,66-4,85)$ & $<0,001$ \\
Obesidad & $2,69(1,39-5,21)$ & 0,003 \\
Transición a la menopausia vs premenopausia & $2,53(1,47-4,34)$ & $<0,001$ \\
Riesgo metabólico muy aumentado & $2,20(1,46-3,32)$ & $<0,001$ \\
Hipertensión arterial & $2,18(1,40-3,40)$ & $<0,001$ \\
Rango etario 45-49 con respecto a 40-44 & $1,89(1,06-3,35)$ & $<0,05$ \\
Sobrepeso & $1,62(1,05-2,50)$ & $<0,05$ \\
\hline
\end{tabular}




\section{FACTORES ASOCIADOS A DOLORES MUSCULARES Y ARTICULARES \\ EN MUJERES AFRODESCENDIENTES CLIMATÉRICAS}

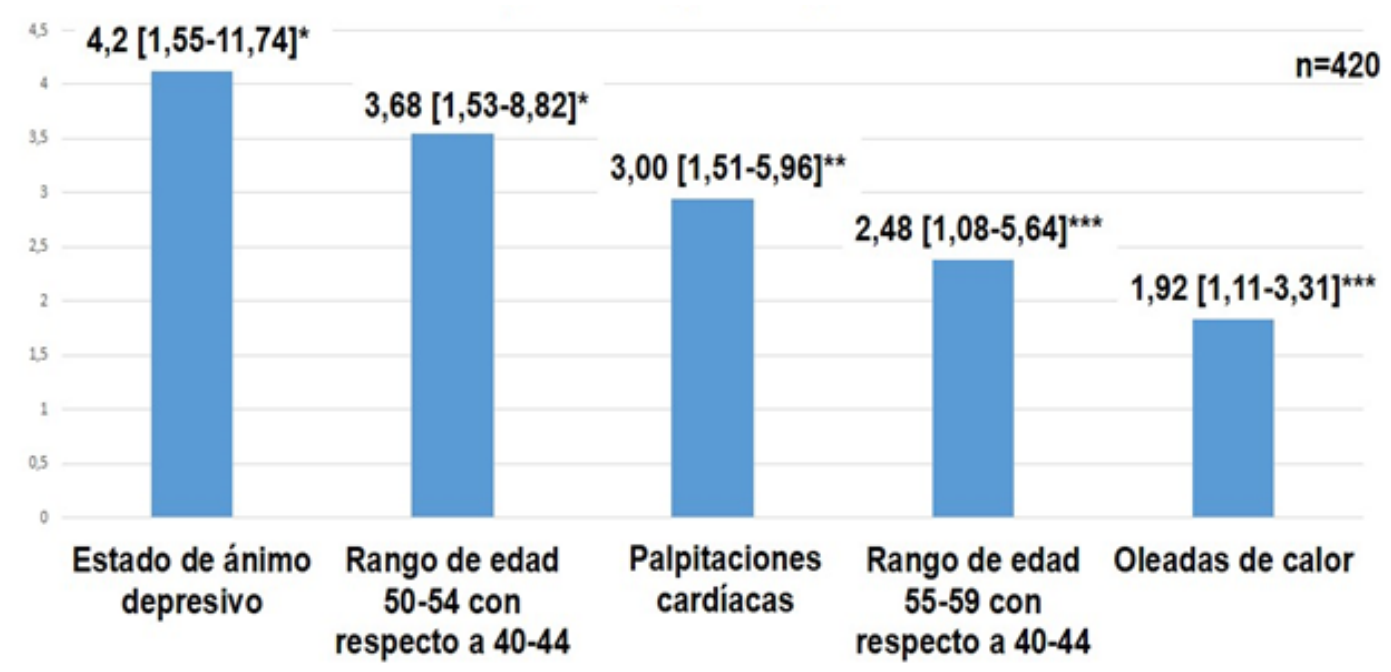

Los datos son presentados en OR [IC95\%]. (") p<0,001. ("*) p=0,001. ("**) p<0,05.

Varias incluidas en el modelo: sequedad vaginal, problemas sexuales, irritabilidad, palpitaciones cardiacas, ansiedad, problemas de sueño, agotamiento fisico/mental, oleadas de calor, rango etario $50-54$ con respecto a 40-44, diabetes mellitus, posmenopausia vs premenopausia, estado de ánimo depresivo, rango etario $55-59$ con respecto a 40-44, obesidad, transición a la menopausia vs premenopausia, hipertensiön arterial, rango etario 45.49 con respecto a $40-44$ y sobrepeso.

Figura 1. Factores asociados a molestias musculoarticulares. Regresión logística ajustada

Tabla 5. Correlación con molestias musculo articulares. Coeficiente de Spearman

\begin{tabular}{lccc}
\hline Variable & rho & IC95\% & $\mathrm{p}$ \\
\hline Perímetro abdominal & 0,322 & 0,233 to 0,405 & \\
Perímetro de la cadera & 0,318 & 0,229 to 0,401 & \\
Edad & 0,212 & 0,119 to 0,302 & $<0,001$ \\
Peso & 0,183 & 0,088 to 0,274 & \\
IMC & 0,173 & 0,078 to 0,264 & \\
Índice cintura/cadera & 0,129 & 0,033 to 0,222 & \\
\hline
\end{tabular}

\section{DISCUSIÓN}

En el presente estudio se encontró que el 57,9\% de un grupo de mujeres afrodescendientes que realizaban sus actividades diarias, informaron DMA, siendo el segundo síntoma más frecuente y antecedido ligeramente $(59,7 \%)$ por las oleadas de calor. Una cifra similar a la nuestra fue informada entre mujeres africanas, en las cuales encontraron 59,0 \% y fue el síntoma menopáusico más frecuente (12). Monterrosa y cols. (3), en una población que incluía mestizas, afrodescendientes e indígenas, encontraron DMA en $72,9 \%$ siendo el síntoma menopaúsico más frecuente. En 8373 mujeres climatéricas latinoamericanas informaron que el 63,0 \% presentaban DMA (13). Chedraui y cols. (14) los identificaron en el 77,2\% de mujeres ecuatorianas, mientras que en mujeres de Malasia, también informaron que los DMA fueron el síntoma menopaúsico más frecuente (15). En mujeres de tres provincias rurales y una urbana de Camboya observaron 79,7 \% (16). Los DMA son un síntoma menopaúsico relatado con importante frecuencia por las mujeres en climaterio, deterioran la calidad de vida y generan carga para el sistema sanitario $(3,12-14$, 16).

Los DMA son un síntoma y al igual que el término artralgia, puede ser descrito como un dolor en una articulación o en varias articulaciones (5). Puede responder a varias condiciones patológicas o a diferentes circunstancias biopsicosociales. Se ha enfatizado en los mecanismos fisiopatológicos que están involucrados en esta sensación dolorosa, especialmente en el papel que cumple la actividad simpática del sistema 
nervioso y en diversos neurotransmisores: serotonina, noradrenalina, sustancia $\mathrm{P}$ y el glutamato, que están relacionados con la nocicepción. Es conocido como las neuronas nociceptoras, regulan la respuesta de las células inmunitarias vasculares, innatas y adaptativas. Al tiempo, que las células inmunes en las terminales nerviosas periféricas y dentro de la médula espinal, liberan mediadores que modulan la sensibilidad (17, $18)$.

Watt (5) entregó una lista de causas de DMA en la perimenopausia: artralgia primaria idiopática $\mathrm{o}$ asociada a la menopausia, osteoartritis, mialgias endocrinas o menopaúsicas, polimialgia reumática, enfermedad ósea de Paget y la fibromialgia, en la cual el dolor DMA se asocia con fatiga, alteración del sueño, ansiedad o depresión y otros síndromes dolorosos como la migraña, el intestino irritable y el dolor torácico atípico. También indica las causas de los DMA o artralgias en la posmenopausia: las primarias o derivadas de la deficiencia estrogénica y las secundarias a alteraciones endocrinológicas, metabólicas, reumatológicas, degenerativas, infecciosas, medicamentosas y oncológicas.

Varios autores $(5,19-22)$ han sugerido la importancia de las hormonas sexuales, especialmente los estrógenos, que es conocido que poseen efectos antiinflamatorios y medianamente inmunosupresores. Estos participan en la regulación del tejido óseo, cartilaginoso y discal, modulando la inflamación y la neurotransmisión del dolor. El 17 $\beta$-estradiol tiene entre sus funciones proteger al hueso de la resorción, entre otros mecanismos, liberando osteoprotegerina, una glicoproteína soluble también conocida como factor inhibidor de la osteoclastogénesis (23). Están identificados receptores estrogénicos en los fibroblastos sinoviales y en los condrocitos articulares, en el hueso subcondral y en los ligamentos, e incluso el estrógeno es sintetizado, por acción de las aromatasas, en los tejidos conectivos con el fin de promover el crecimiento del cartílago o prevenir su degradación (5). Se ha señalado que existe relación inversa entre los estrógenos y la degradación del cartílago (21). También se observó elevación del marcador de destrucción del colágeno tipo-II (CTX-II) en la orina de ratas castradas, y la posterior reducción al administrar estrógenos exógenos (24). Los estrógenos, actúan sobre los monocitos y los macrófagos regulando hacia abajo la IL-1, IL-6 y FNT- $\alpha$, citoquinas que inhiben la formación de proteoglicanos y la formación de hueso nuevo, que promueven inflamación sinovial e inducen resorción del cartílago $(5,13)$.

Se ha señalado que la deficiencia estrogénica que se produce dentro del climaterio y especialmente en la posmenopausia, pudiese participar en el establecimiento de diferentes patologías esqueléticas, incluidas la osteoartritis y la fibromialgia. La primera, es la forma más común de artritis, afecta el cartílago articular, el hueso, la membrana sinovial y los ligamentos, generando los clásicos "osteofitos" en la radiografía y causando dolor articular, hinchazón y pérdida de función. Es la razón más común para la necesidad de realizar reemplazo articular. La segunda, es una entidad poco comprendida, anteriormente conocida como el síndrome del dolor miofascial, en la cual es probable que influencias genéticas y hormonales, sean importantes para generar mayor sensibilidad al dolor y tendencia a la presencia de otros cuadros de dolor crónico $(5,22,25)$. Cuando las mujeres informan DMA, no necesariamente son indicativas de alguno de estos dos diagnósticos. El síntoma no se debe subvalorar, lo correcto será realizar los estudios clínicos o complementarios e intentar establecer un diagnóstico para realizar un tratamiento etiológico.

Mohamadzade y cols. (21) observaron que las mujeres posmenopáusicas con niveles reducidos de estradiol, informaron más síntomas menopaúsicos severos y tasas más altas de DMA. Una de las explicaciones propuestas es que la reducción estrogénica se relaciona con la descomposición de proteínas de la matriz del colágeno, a través de las metaloproteinasas producidas 


\section{FACTORES ASOCIADOS A DOLORES MUSCULARES Y ARTICULARES \\ EN MUJERES AFRODESCENDIENTES CLIMATÉRICAS}

por los osteoclastos (26). Sowers y cols. (27) señalaron la asociación significativa entre el bajo nivel de estradiol o de la 2-hidroxiestrona y la osteoartritis de rodilla. $\mathrm{El}$ antecedente de ciclos menstruales extremadamente irregulares y/o los bajos niveles de 17 $\beta$-estradiol, se han relacionado con fenómenos inflamatorios articulares en mujeres en peri- o posmenopausia $(16,21)$. También se ha anotado que las mujeres en climaterio que anteriormente habían utilizado anticonceptivos orales, tuvieron menor frecuencia de DMA 16,1 \% vs 8,7 \%; OR: 0,50 (IC95 \%: 0,360,69) (13). Barnabei y cols. (28), en un ensayo clínico que hace parte del Women's Health Initiative (WHI), encontraron que la administración de estrógenos conjugados más acetato de medroxiprogesterona con respecto a placebo, conllevó mayor alivio del dolor y de la rigidez articular.

Los esteroides sexuales femeninos poseen mecanismos de acción complejos, aún se ameritan estudios para establecer el papel real que pueden tener en la génesis o en la perpetuación de los DMA y en diversas patologías que tienen la capacidad de generarlos (19, 20). Es aceptado que la disminución estrogénica se relaciona con el envejecimiento y con el deterioro psicológico del climaterio, no obstante no existe suficiente información para sugerir la administración de terapia hormonal en el manejo de los DMA (5).

Algunos factores sociales se han utilizado para explicar los DMA. Olaolorun y Lawoyin (12) encontraron que el esfuerzo físico que debían realizar las mujeres para obtener servicios sociales básicos y la realización de tareas domésticas sin ayuda, se relacionaron con DMA.

En la presente serie se encontró que todos los síntomas menopaúsicos explorados con la escala MRS, excepto los urinarios, se asociaron a DMA en el análisis bivariado. A su vez, el estado de ánimo depresivo, las palpitaciones cardiacas y las oleadas de calor, lo hicieron cuatro, tres y dos veces respectivamente, en el modelo ajustado. Resultados similares fueron encontrados en una población multiétnica latinoamericana (13). También son coherentes con los observados en mujeres nigerianas (12). Generalmente los síntomas menopaúsicos son consecuencia del declinar de la función ovárica, pero la presencia y severidad suele variar según aspectos geográficos, socioculturales, estilo de vida y étnico/raciales (16). Por ello, en cada comunidad de mujeres se deben conocer las cifras de frecuencia y tenerlo en cuenta al momento de interrogarlas dentro de la atención sanitaria. Es posible que el déficit de estrógenos que caracteriza al climaterio, interactuando en conjunto con varios neurotransmisores, neuropéptidos y neuroesteroides, sean el común denominador que explique la relación existente entre todos los síntomas menopaúsicos, como fue observado en el presente estudio $(5,14)$.

Blümel y cols. (13) encontraron que la menopausia prematura, el nivel educativo y socioeconómico, se asociaron con DMA. Con los resultados de este estudio se agregan: los rangos etarios mayores, los estados menopáusicos más avanzados, la obesidad, el riesgo metabólico muy aumentado, la diabetes y la hipertensión arterial. Thapa y Yang (16) observaron que la historia de artritis reumatoide, se asoció con peores puntuaciones de calidad de vida $(\beta=0.195, p=0.004)$. Además, indicaron una tendencia creciente de la edad con varios síntomas menopaúsicos, incluidos los DMA, en mujeres climatéricas del Sudeste asiático. Los autores han observado similar tendencia entre las mediciones antropométricas y la edad, con los DMA.

Karacan (29) indicó que la obesidad y el sobrepeso están frecuentemente relacionadas con el sedentarismo y recomiendan la actividad física para disminuir los síntomas somáticos de la menopausia. En las afrodescendientes estudiadas, ambas se asociaron con DMA, aunque perdieron significancia en el modelo ajustado. El ejercicio físico se ha indicado para tratar y prevenir afecciones en las mujeres de mediana edad, 
tanto psicológicas como el DMA $(5,30)$. Hacen falta estudios para explicar la asociación observada entre DMA con la diabetes y el elevado riesgo metabólico. Existen importantes vacíos sobre los DMA en las mujeres en menopausia. Se debe considerar como un importante interrogante a dilucidar en futuros estudios, si los DMA son expresiones sintomáticas propias del incremento de la edad o son manifestaciones de patologías óseas, articulares o musculares, que no han sido plenamente identificadas o diagnosticadas (5).

El estudio contribuye a visibilizar en mejor forma la existencia de los DMA. Pretende poner en perspectiva la necesidad de que los DMA sean valorados dentro de los síntomas menopaúsicos y busca generar conciencia en la necesidad de que sean suficientemente interrogados en la práctica clínica rutinaria. La frecuencia observada exige, la realización de estudios con otros diseños que permitan dimensionar en mejor forma las implicaciones que tienen los DMA, que en otros escenarios clínicos distintos a los de la valoración del climaterio, son explorados bajo el término de artralgias. Posee las limitaciones propias de los estudios transversales. No abordó consideraciones sociales o estilos de vida, aspectos nutricionales y actividad física, que pueden modular la frecuencia y severidad de los DMA. Es posible que algunos datos puedan estar sobredimensionados o infradimensionados, a consecuencia de sesgos de selección que no se pudieron controlar. Se recomienda a los entes gubernamentales y no gubernamentales, que dictan políticas de atención en salud, crear programas que exploren suficientemente los síntomas que refieren las mujeres en etapa vital de climaterio. Los profesionales de la salud, sobre todo los de atención primaria que atienden mujeres en climaterio, deben interrogar adecuadamente a sus pacientes sobre la existencia y severidad de todos los síntomas menopáusicos y no solos las oleadas de calor o el síndrome genitourinario de la menopausia. Además todos los profesionales de la salud deben ilustrar a las mujeres sobre la importancia que tienen los DMA, incentivándolas a manifestarlo en caso de estar presentes y ponerlos entre el listado de los motivos de consulta. Se subraya que los DMA informados por las mujeres en el climaterio, son básicamente síntomas que deben ser adecuadamente estudiados con herramientas clínicas y/o paraclínicas, para tratar de identificar los potenciales estados mórbidos que puedan estar subyacentes.

Los autores concluyen que en un grupo de mujeres afrodescendientes en etapa vital de climaterio, que adelantaban actividades cotidianas y residían en la región caribe colombiana, se observó elevada frecuencia de DMA. El envejecimiento (establecido por rangos etarios), síntomas psicológicos como el estado de ánimo depresivo y la sintomatología somato/vegetativa, específicamente las oleadas de calor y las palpitaciones cardiacas, se asociaron significativamente con la sintomatología dolorosa muscular y articular. Se sugiere que los DMA hagan parte del interrogatorio clínico rutinario que se realiza a las mujeres que están en menopausia y climaterio.

\section{Sin conflictos de interés.}

\section{REFERENCIAS}

1. Larroy C, Quiroga-Garza A, González-Castro PJ, Robles Sánchez JI. Symptomatology and quality of life between two populations of climacteric women. Arch WomensMent Health. 2020; 23(4):517-525. doi:10.1007/s00737-019-01005-y

2. El Khoudary SR, Greendale G, Crawford SL, Avis NE, Brooks MM, Thurston RC, et al. The menopause transition and women's health at midlife: a progress report from the Study of Women's Health Across the Nation (SWAN). Menopause. 2019; 26(10):1213-1227. doi:10.1097/GME.0000000000001424

3. Monterrosa-Castro A, Romero-Pérez I, PaterninaCaicedo Á. Manifestaciones músculo-articulares, y no las oleadas de calor, es el síntoma más prevalente en mujeres climatéricas del Caribe colombiano. SaludUninorte, Barranquilla [Internet]. 2010 [consultado 1 de mayo de 2021]; 26(2):179-188. Disponible en: http://www.scielo.org.co/scielo.php?script=sci arttext\&pid=S0120-55522010000200002\&lng=en 


\section{FACTORES ASOCIADOS A DOLORES MUSCULARES Y ARTICULARES EN MUJERES AFRODESCENDIENTES CLIMATÉRICAS}

4. Heinemann K, Ruebig A, Potthoff P, Schneider HP, Strelow F, Heinemann LA, et al. The Menopause Rating Scale (MRS) scale: a methodological review. Health Qual Life Outcomes. 2004; 2:45. doi:10.1186/14777525-2-45

5. Watt FE. Musculoskeletal pain and menopause. Post Reprod Health. 2018; 24(1):34-43. doi: $10.1177 / 2053369118757537$

6. United Nations [Internet]. Nueva York: International Decade for People of African Descent. 2015-2024 [consultado 1 de mayo de 2021]. Disponible en: https:// www.un.org/en/observances/decade-people-africandescent

7. World Bank [Internet]. Washington DC: Afrodescendants in Latin America: Toward a Framework of Inclusion. 2018 [consultado 1 de mayo de 2021]. Disponible en: https://openknowledge.worldbank.org/ handle/10986/30201?locale-attribute=en

8. Departamento Administrativo de Estadística de Colombia (DANE) [Internet]. Bogotá: Población negra, afrocolombiana, raizal y palenquera; 2019 [consultado 1 de mayo de 2021]. Disponible en: https:/www.dane. gov.co/files/investigaciones/boletines/grupos-etnicos/ presentacion-grupos-etnicos-poblacion-NARP-2019. pdf

9. Monterrosa A, Herazo E, Oviedo HC, Campo A. Utilidad del Menopause Rating Scale (MRS) en indígenas colombianas en climaterio. Duazary. 2017; 14(1):8-15. doi:10.21676/2389783X.1737

10. Harlow SD, Gass M, Hall JE, Lobo R, Maki P, Rebar RW, et al.; STRAW + 10 Collaborative Group. Executive summary of the Stages of Reproductive Aging Workshop + 10: addressing the unfinished agenda of staging reproductive aging. J Clin Endocrinol Metab. 2012; 97(4):1159-1168. doi:10.1210/jc.20113362

11. Buendía R, Zambrano M, Díaz A, Reino A, Ramírez J, Espinosa E. Puntos de corte de perímetro de cintura para el diagnóstico de obesidad abdominal en población colombiana usando bioimpedanciometría como estándar de referencia. Rev Colomb Cardiol. 2016; 23(1):19-25. doi.org/10.1016/j.rccar.2015.07.011

12. Olaolorun FM, Lawoyin TO. Experience of menopausal symptoms by women in an urban community in Ibadan, Nigeria. Menopause. 2009; 16(4):822-830. doi:10.1097/gme.0b013e318198d6e7

13. Blümel JE, Chedraui P, Baron G, Belzares E, Bencosme A, Calle A, et al. Menopause could be involved in the pathogenesis of muscle and joint aches in mid-aged women. Maturitas. 2013; 75(1):94-100. doi:10.1016/j. maturitas.2013.02.012

14. Chedraui P, Aguirre W, Hidalgo L, Fayad L. Assessing menopausal symptoms among healthy middle aged women with the Menopause Rating Scale. Maturitas. 2007; 57(3):271-278. doi:10.1016/j. maturitas.2007.01.009

15. Rahman SA, Zainudin SR, Mun VL. Assessment of menopausal symptoms using modified Menopause Rating Scale (MRS) among middle age women in Kuching, Sarawak, Malaysia. Asia Pac Fam Med. 2010; 9(1):5. doi:10.1186/1447-056X-9-5

16. Thapa R, Yang Y. Menopausal symptoms and related factors among Cambodian women. Women Health. 2020; 60(4):396-411. doi:10.1080/03630242.2019.16 43815

17. Pinho-Ribeiro FA, Verri WA Jr, Chiu IM. Nociceptor Sensory Neuron-Immune Interactions in Pain and Inflammation. Trends Immunol. 2017; 38(1):5-19. doi:10.1016/j.it.2016.10.001

18. Chen O, Donnelly CR, Ji RR. Regulation of pain by neuro-immune interactions between macrophages and nociceptor sensory neurons. Curr Opin Neurobiol. 2020; 62:17-25. doi:10.1016/j.conb.2019.11.006

19. Craft RM. Modulation of pain by estrogens. Pain. 2007; 132(Suppl-1):S3-S12. doi:10.1016/j.pain.2007.09.028

20. Martin VT. Ovarian hormones and pain response: a review of clinical and basic science studies. Gend Med. 2009; 6(Suppl-2):168-192. doi:10.1016/j. genm.2009.03.006

21. Mohamadzade M, Sadatmahalleh SJ, Ziaei S, Zaeemzadeh N, Kazemnejad A. The relationship between musculoskeletal pains, serum estradiol level and climacteric symptoms in postmenopausal women. 17 June 2020, Preprint (Version 1). Disponible en: in Research Square doi.org/10.21203/rs.3.rs-34791/v1

22. Karsdal MA, Bay-Jensen AC, Henriksen K, Christiansen C. The pathogenesis of osteoarthritis involves bone, cartilage and synovial inflammation: may estrogen be a magic bullet? Menopause Int. 2012; 18(4):139-146. doi:10.1258/mi.2012.012025

23. Rogers A, Saleh G, Hannon RA, Greenfield D, Eastell R. Circulating estradiol and osteoprotegerin as determinants of bone turnover and bone density in postmenopausal women. J Clin Endocrinol Metab. 2002; 87(10):4470-4475. doi:10.1210/jc.2002-020396 
24. Linn S, Murtaugh B, Casey E. Role of sex hormones in the development of osteoarthritis. PMR. 2012; 4(5-Suppl):S169-173. doi:10.1016/j.pmrj.2012.01.013

25. Hussain SM, Cicuttini FM, Alyousef B, Wang Y. Female hormonal factors and osteoarthritis of the knee, hip and hand: a narrative review. Climacteric. 2018; 21(2):132-139. doi:10.1080/13697137.2017.1421926

26. Kapila S, Wang W, Uston K. Matrix metalloproteinase induction by relaxin causes cartilage matrix degradation in target synovial joints. Ann N Y Acad Sci. 2009; 1160:322-328. doi:10.1111/j.1749-6632.2009.03830.x

27. Sowers MR, McConnell D, Jannausch M, Buyuktur AG, Hochberg M, Jamadar DA. Estradiol and its metabolites and their association with knee osteoarthritis. Arthritis Rheum. 2006; 54:2481-7. doi.org/10.1002/art.22005

28. Barnabei VM, Cochrane BB, Aragaki AK, Nygaard I ,Williams R, Stan M. et al. Menopausal symptoms and treatment-related effects of estrogen and progestin in the Women's Health Initiative. Obstet Gynecol. 2005; 105(5Pt1):1063-1073. doi:10.1097/01.AOG. 0000158120.47542 .18

29. Karacan S. Effects of long-term aerobic exercise on physical fitness and postmenopausal symptoms with menopausal rating scale. Science \& Sports. 2010; 25:39-46. doi.org/10.1016/j.scispo.2009.07.004

30. Dugan SA. Exercise for health and wellness at midlife and beyond: balancing benefits and risks. Phys Med Rehabil Clin N Am. 2007; 18(3):555-xi. doi:10.1016/j. pmr.2007.05.006 